\title{
Heat transfer analysis in a second grade fluid over and oscillating vertical plate using fractional Caputo-Fabrizio derivatives
}

\author{
Nehad Ali Shah ${ }^{1}$, Ilyas Khan ${ }^{2, a}$ \\ ${ }^{1}$ Abdus Salam School of Mathematical Sciences, GC University, Lahore, Pakistan \\ 2 Basic Sciences Department, College of Engineering Majmaah University, P.O. Box 66, Majmaah 11952, Saudi Arabia
}

Received: 29 April 2016 / Accepted: 30 May 2016 / Published online: 1 July 2016

(C) The Author(s) 2016. This article is published with open access at Springerlink.com

\begin{abstract}
This paper presents a Caputo-Fabrizio fractional derivatives approach to the thermal analysis of a second grade fluid over an infinite oscillating vertical flat plate. Together with an oscillating boundary motion, the heat transfer is caused by the buoyancy force induced by temperature differences between the plate and the fluid. Closed form solutions of the fluid velocity and temperature are obtained by means of the Laplace transform. The solutions of ordinary second grade and Newtonian fluids corresponding to time derivatives of integer and fractional orders are obtained as particular cases of the present solutions. Numerical computations and graphical illustrations are used in order to study the effects of the Caputo-Fabrizio time-fractional parameter $\alpha$, the material parameter $\alpha_{2}$, and the Prandtl and Grashof numbers on the velocity field. A comparison for time derivative of integer order versus fractional order is shown graphically for both Newtonian and second grade fluids. It is found that fractional fluids (second grade and Newtonian) have highest velocities. This shows that the fractional parameter enhances the fluid flow.
\end{abstract}

\section{Introduction}

The concept of fractional-order derivatives is as old as for integer-order derivatives. For the past three decades, this subject was limited only to mathematics. However, in the last few years, the concepts of fractional calculus were frequently applied to other disciplines. Recently, this subject has been extended in various directions such as fractional-order multipoles in electromagnetism, electrochemistry, tracer in fluid flows, model of neurons in biology, finance, signal processing, applied mathematics, bio-engineering, viscoelasticity, fluid mechanics, and fluid dynamics [1]. In fluid dynamics, the fractional derivative models were used widely in the

\footnotetext{
a e-mail: i.said@mu.edu.sa
}

past for the study of viscoelastic materials such as polymers in the glass transition and in the glassy state [2]. Recently, it has increasingly been seen as an efficient tool through which a useful generalization of physical concepts can be obtained. The fractional derivatives used most are the Riemann-Liouville fractional derivative and the Caputo fractional derivative $[3,4]$. It is well known that these operators exhibit difficulties in applications. For example, the Riemann-Liouville derivative of a constant is not zero and the Laplace transform of the Riemann-Liouville derivative contains terms without physical signification. The Caputo fractional derivative has eliminated these difficulties, but the kernel of the definition is a singular function. Caputo and Fabrizio have introduced recently a new definition of the fractional derivatives with an exponential kernel without singularities [5]. The Caputo-Fabrizio temporal-fractional derivative is suitable in the use of the Laplace transform. The spatial representation of the Caputo-Fabrizio derivative is adequate in the use of the Fourier transform. Due to increased interest in modeling with the help of the fractional derivative, several existing fluid models are generalized and fractional derivatives models have been developed. Amongst them, the most popular fluid models are fractional second grade model, the fractional Maxwell model, the fractional Oldroyd-B model, the fractional Burgers model, etc. The second grade fluid model is a sub-model of differential type fluids whereas the other models (Maxwell, Oldroyd, and Burgers) form a subclass of rate-type fluids. According to Tan and Mingyu [6], the starting point of fractional derivative model of nonNewtonian fluids is usually a classical non-Newtonian model, which is modified by replacing the time derivative of an integer order by Riemann-Liouville fractional derivative i.e. a time derivative of fractional order. In the earlier studies, Friedrich [7] generalized the ordinary Maxwell fluid model to the fractional Maxwell model and studied it as a function of the relaxation and retardation times. Tan et al. [8] addressed in a short note unsteady flows of a viscoelastic 
fluid with a fractional Maxwell model between two parallel plates. Hayat et al. [9] studied periodic unidirectional flows of a viscoelastic fluid with the fractional Maxwell model. Yin and Zhu [10] examined an oscillating flow of a viscoelastic fluid in a pipe with the fractional Maxwell model. Qi and Jin [11] investigated unsteady rotating flows of viscoelastic fluid with the fractional Maxwell model between coaxial cylinders. Qi and Xu [12] analyzed an unsteady channel flow of a viscoelastic fluid with fractional Maxwell model. Mahmood et al. [13] determined the velocity field and the associated shear stress corresponding to the torsional oscillatory flow of a generalized Maxwell fluid between two infinite coaxial circular cylinders by means of the Laplace and Hankel transforms. Fetecau et al. [14] established exact solutions for the flow of a fractional Maxwell fluid between coaxial cylinders. Jamil et al. [15] provided new exact solutions for Stokes' first problem of the Maxwell fluid using a fractional derivative approach. Similar attempts for other viscoelastic fluids, namely the Oldroyd-B fluid, the Burgers fluid, and the generalized Burgers fluid were made by Khan et al. [1618], Fetecau et al. [19], Qi and Jin [20], Jamil et al. [21], Zheng et al. [22], Liu et al. [23], Tong [24], and Zheng et al. [25]. Apart from the rate-type fluids, the idea of fractional derivatives is also implemented on differential type fluids, particularly the second grade fluid; see for instance [26-29]. In view of the above discussion, although some important investigations in the past on fractional models of a non-Newtonian fluid were carried out, yet this field is not as rich as for integer derivatives of non-Newtonian fluids problems. Therefore, very limited investigations were carried out for fractional models of non-Newtonian fluids, more exactly for exact solutions. Such investigations are even more scarce when the non-Newtonian fluid flow is considered in the presence of convection heat transfer. With this motivation, Vieru et al. [30] in a recent investigation used the idea of a fractional derivative and studied the free convection flow of a viscous fluid past a vertical infinite plate with Newtonian heating and constant mass diffusion conditions. However, such investigations are not available for any subclass of non-Newtonian fluids. More exactly, there is no single investigation available on a fractional model of a non-Newtonian fluid together with convection heat transfer where the exact solutions are established. Therefore, the present work aims to make such an attempt. For this purpose a simpler subclass of differential type fluid, known as a second grade fluid, is chosen. More exactly, the second grade fluid in the presence of heat transfer analysis due to buoyancy convection over an oscillating vertical plate is investigated by using the fractional derivative approach. The above mentioned papers, which have considered flows modeled by fractional differential equations, are based on Riemann-Liouville or Caputo fractional derivative operators. For this reason, analytical solutions are expressed as a series of generalized functions
(Mittag-Leffler, Lorenzo-Hartley, Robotnov) making them inadequate for the numerical calculation. In this paper we consider the governing equation of the flow as a fractional differential equation based on the new fractional derivative without singular kernel. By using this derivative, calculations become simpler and the final form of the solutions is suitable for numerical calculations.

The rest of the paper is arranged as follows. The mathematical formulation of the problem is given in Sect. 2. Exact solutions via the Laplace transform are established in Sect. 2 followed by some limiting cases in Sect. 3. Graphical results are presented in Sect. 4 for important pertinent parameters. This paper ends with some important conclusions in Sect. 5. Some important formulas used in this paper are presented in the appendix.

\section{Mathematical formulation and solution of the problem}

Let us consider an incompressible second grade fluid lying over an infinite rigid flat plate occupying the $x y$ plane. The $y$ axis is taken normal to the plate. Initially the fluid and the plate are at rest and its temperature is $T_{\infty}$ (ambient fluid temperature). After time $t=0^{+}$, the plate begins to oscillate in its own plane and induces the motion in the fluid with velocity $f H(t) \exp (i \omega t)$, where $H(t)$ is the unit step function, $f$ is the constant having the dimension of a velocity and $\omega$ is the frequency of the oscillation. At the same time, the plate temperature is raised to $T_{w}$ (wall temperature) which is thereafter kept constant. We assume that the velocity and temperature are functions of $y$ and $t$ only. For such a flow, the constraint of incompressibility is identically satisfied. Taking the usual Boussinesq approximation, the unsteady flow is governed by the following set of partial differential equations [31]:

$$
\begin{aligned}
\frac{\partial u(y, t)}{\partial t}= & v \frac{\partial^{2} u(y, t)}{\partial y^{2}}+\frac{\alpha_{1}}{\rho} \frac{\partial^{3} u(y, t)}{\partial y^{2} \partial t} \\
& +g \beta_{T}\left(T(y, t)-T_{\infty}\right) ; y, \quad t>0, \\
\frac{\partial T(y, t)}{\partial t}= & \frac{k}{\rho C_{p}} \frac{\partial^{2} T(y, t)}{\partial y^{2}} ; y, t>0,
\end{aligned}
$$

where $u(y, t)$ is the fluid velocity, $T(y, t)$ is the fluid temperature, $v$ is the kinematic viscosity of the fluid, $\rho$ is the constant density, $\alpha_{1}$ is the second grade parameter, $g$ is the gravitational acceleration, $\beta_{T}$ is the volumetric coefficient of thermal expansion, $C_{p}$ is the heat capacity at constant pressure, and $k$ is the thermal conductivity.

The appropriate initial and boundary conditions are

$u(y, 0)=0, \quad T(y, 0)=T_{\infty} ; \quad y>0$, 
$u(0, t)=f H(t) \exp (i \omega t), T(0, t)=T_{w}, t>0, f>0$,

$u(y, t) \rightarrow 0, \quad T(y, t) \rightarrow T_{\infty}, \quad$ as $y \rightarrow \infty, \quad t>0$.

Introducing the following dimensionless variables into Eqs. (1)-(5):

$t^{*}=\frac{f^{2} t}{v}, \quad y^{*}=\frac{f y}{v}, \quad u^{*}=\frac{u}{f}, \quad \theta=\frac{T-T_{\infty}}{T_{w}-T_{\infty}}$,

$\alpha_{2}=\frac{\alpha_{1} f^{2}}{\mu \nu}, \quad \mathrm{Gr}=\frac{\nu g \beta_{T}\left(T_{w}-T_{\infty}\right)}{f^{3}}, \quad \operatorname{Pr}=\frac{\mu C_{p}}{k}$,

and dropping the star notation, we obtain the dimensionless problem

$\frac{\partial u(y, t)}{\partial t}=\frac{\partial^{2} u(y, t)}{\partial y^{2}}+\alpha_{2} \frac{\partial^{3} u(y, t)}{\partial y^{2} \partial t}+\operatorname{Gr} \theta(y, t)$,

$\frac{\partial \theta(y, t)}{\partial t}=\frac{1}{\operatorname{Pr}} \frac{\partial^{2} \theta(y, t)}{\partial y^{2}}$,

$u(y, 0)=0, \quad \theta(y, 0)=0, \quad y \geq 0$,

$u(0, t)=H(t) \exp (i \omega t), \quad \theta(0, t)=1, t>0$,

$u(y, t) \rightarrow 0, \quad \theta(y, t) \rightarrow 0$ as $y \rightarrow \infty, t \geq 0$.

In order to develop a model with time-fractional derivatives, we replace the time derivative of order one with the CaputoFabrizio time-fractional derivative of order $\alpha \in[0,1]$, and Eqs. (7) and (8) are written as

$D_{t}^{\alpha} u(y, t)=\frac{\partial^{2} u(y, t)}{\partial y^{2}}+\alpha_{2} D_{t}^{\alpha} \frac{\partial^{2} u(y, t)}{\partial y^{2}}+\operatorname{Gr} \theta(y, t)$,

$\operatorname{Pr} D_{t}^{\alpha} T(y, t)=\frac{\partial^{2} T(y, t)}{\partial y^{2}}$

where the Caputo-Fabrizio time-fractional derivative [5] is defined by

$$
\begin{aligned}
D_{t}^{\alpha} u(y, t) & =\frac{1}{1-\alpha} \int_{0}^{t} \exp \left(\frac{-\alpha(t-\tau)}{1-\alpha}\right) u^{\prime}(\tau) \mathrm{d} \tau, \\
\alpha & \in[0,1] .
\end{aligned}
$$

\subsection{Calculation for temperature}

Applying the Laplace transform to Eqs. (13), (10) $2,(11)_{2}$, and using $(9)_{2}$, we obtain
$\operatorname{Pr} \frac{s}{(1-\alpha) s+\alpha} \bar{\theta}(y, s)=\frac{\partial^{2} \bar{\theta}(y, s)}{\partial y^{2}}$,

$\bar{\theta}(0, s)=\frac{1}{s}, \quad \bar{\theta}(y, s) \rightarrow 0 \quad$ as $\quad y \rightarrow \infty$.

Using the notation $\gamma=\frac{1}{1-\alpha}$, we have

$\frac{\operatorname{Pr} \gamma s}{s+\alpha \gamma} \bar{\theta}(y, s)=\frac{\partial^{2} \bar{\theta}(y, s)}{\partial y^{2}}$,

$\bar{\theta}(0, s)=\frac{1}{s}, \quad \bar{\theta}(y, s) \rightarrow 0$ as $y \rightarrow \infty$.

The solution of the problem (15), (16) is

$\bar{\theta}(y, s)=\frac{1}{s} \exp \left(-y \sqrt{\frac{\operatorname{Pr} \gamma s}{s+\alpha \gamma}}\right)=\Phi(y, s ; \operatorname{Pr} \gamma, \alpha \gamma)$,

respectively, and the temperature field is given by

$\theta(y, t)=\varphi(y, t ; \operatorname{Pr} \gamma, \alpha \gamma), \quad 0<\alpha<1$,

where functions $\Phi(y, s ; a, b)$ and $\varphi(y, t ; a, b)$ are defined by (A1), (A2) from the appendix.

2.2 Temperature field for the ordinary case $(\alpha=1)$

The temperature expression corresponding to the ordinary case is obtained based on a property of the Caputo-Fabrizio fractional derivative, namely

$$
\begin{aligned}
\theta(y, t) & =\lim _{\alpha \rightarrow 1} \varphi(y, t ; \operatorname{Pr} \gamma, \alpha \gamma)=\lim _{\gamma \rightarrow \infty} \varphi(y, t ; \operatorname{Pr} \gamma, \alpha \gamma) \\
& =1-\frac{2}{\pi} \int_{0}^{\infty} \frac{\sin (y x)}{x} \exp \left(-\frac{t x^{2}}{\operatorname{Pr}}\right) \mathrm{d} x .
\end{aligned}
$$

Using the formula [32]

$\int_{0}^{\infty} \frac{\sin (b x)}{x} \exp \left(-a x^{2}\right) \mathrm{d} x=\frac{\pi}{2} \operatorname{erf}\left(\frac{b}{2 \sqrt{a}}\right)$

we obtain

$\theta(y, t)=1-\operatorname{erf}\left(\frac{y \sqrt{\operatorname{Pr}}}{2 \sqrt{t}}\right)=\operatorname{erfc}\left(\frac{y \sqrt{\operatorname{Pr}}}{2 \sqrt{t}}\right)$. 


\subsection{Calculation for velocity field}

Applying the Laplace transform to Eq. (12) and using the initial and boundary conditions (9)-(11), we obtain the problem

$\frac{\gamma s}{s+\alpha \gamma} \bar{u}(y, s)=\frac{\partial^{2} \bar{u}(y, s)}{\partial y^{2}}+\alpha_{2} \frac{\gamma s}{s+\alpha \gamma} \frac{\partial^{2} \bar{u}(y, s)}{\partial y^{2}}$ $+\operatorname{Gr} \bar{\theta}(y, s)$,

$\bar{u}(y, s)=\frac{1}{s-i \omega}, \quad \bar{u}(y, s) \rightarrow 0$ as $y \rightarrow \infty$.

Using Eq. (17) in Eq. (22) and rearranging, we obtain

$$
\begin{aligned}
& \gamma s \bar{u}(y, s)=\left[\left(1+\alpha_{2} \gamma\right) s+\alpha \gamma\right] \frac{\partial^{2} \bar{u}(y, s)}{\partial y^{2}} \\
& +\operatorname{Gr} \frac{(s+\gamma)}{s} \exp \left(-y \sqrt{\frac{\operatorname{Pr} \gamma s}{s+\alpha \gamma}}\right),
\end{aligned}
$$

$\bar{u}(y, s)=\frac{1}{s-i \omega}, \quad \bar{u}(y, s) \rightarrow 0$ as $y \rightarrow \infty$.

The solution of the partial differential equation (24) with the conditions (25) is

$$
\begin{aligned}
\bar{u}(y, s)= & \frac{1}{s-i \omega} \exp \left(-y \sqrt{\frac{a_{1} s}{s+a_{2}}}\right) \\
& -d_{1} \frac{1}{s} \exp \left(-y \sqrt{\frac{a_{1} s}{s+a_{2}}}\right) \\
& -d_{2} \frac{1}{s^{2}} \exp \left(-y \sqrt{\frac{a_{1} s}{s+a_{2}}}\right) \\
& -d_{3} \frac{1}{s+b_{2}} \exp \left(-y \sqrt{\frac{a_{1} s}{s+a_{2}}}\right) \\
& +d_{1} \frac{1}{s} \exp \left(-y \sqrt{\frac{\operatorname{Pr} \gamma s}{s+\alpha \gamma}}\right) \\
& +d_{2} \frac{1}{s} \exp \left(-y \sqrt{\frac{\operatorname{Pr} \gamma s}{s+\alpha \gamma}}\right) \\
& +d_{3} \frac{1}{s+b_{2}} \exp \left(-y \sqrt{\frac{\operatorname{Pr} \gamma s}{s+\alpha \gamma}}\right),
\end{aligned}
$$

where $a_{1}=\frac{\gamma}{1+\alpha_{2} \gamma}, a_{2}=\alpha a_{1}, b_{1}=\frac{\mathrm{Gr}}{\gamma\left(1-\operatorname{Pr}-\alpha_{2} \gamma \operatorname{Pr}\right)}, b_{2}=$ $\frac{\alpha \gamma(1-\operatorname{Pr})}{\left(1-\operatorname{Pr}-\alpha_{2} \gamma \operatorname{Pr}\right)}, d_{1}=\frac{\operatorname{Gr}\left[(1-\operatorname{Pr})+\alpha_{2} \gamma \operatorname{Pr}\right]}{\gamma(1-\operatorname{Pr})^{2}}, d_{2}=\frac{\operatorname{Gr} \alpha}{(1-\operatorname{Pr})}, d_{3}=$ $\frac{\mathrm{Gr}}{(1-\operatorname{Pr})^{2}} \frac{\alpha_{2}^{2} \operatorname{Pr}^{2} \gamma}{(1-\operatorname{Pr})-\alpha_{2} \gamma \operatorname{Pr}}, \operatorname{Pr} \neq 1$.

Now, using the formulas from the appendix, we obtain the velocity field

$u(y, t)=U_{1}(y, t)+U_{2}(y, t)+\psi\left(y, t ; a_{1}, a_{2}, i \omega\right)$, where

$$
\begin{aligned}
U_{1}(y, t)= & \left(1-d_{1}-d_{3}\right) \varphi\left(y, t ; a_{1}, a_{2}\right) \\
& +\left(d_{1}+d_{3}\right) \varphi(y, t ; \operatorname{Pr} \gamma, \alpha \gamma) \\
& +d_{3}\left[\psi\left(y, t ; \operatorname{Pr} \gamma, \alpha \gamma,-b_{2}\right)\right. \\
& \left.-\psi\left(y, t ; a_{1}, a_{2},-b_{2}\right)\right] \\
U_{2}(y, t)= & d_{2} \int_{0}^{t}\left[\varphi(y, \tau ; \operatorname{Pr} \gamma, \alpha \gamma)-\varphi\left(y, \tau ; a_{1}, a_{2}\right)\right] \mathrm{d} \tau .
\end{aligned}
$$

In order to obtain the real part and the imaginary part, we observe that

$$
\begin{aligned}
\psi\left(y, s ; a_{1}, a_{2}, i \omega\right)= & -\omega \frac{\omega}{s^{2}+\omega^{2}} \Phi\left(y, s ; a_{1}, a_{2}\right) \\
& +i \omega \frac{\omega}{s^{2}+\omega^{2}} \Phi\left(y, s ; a_{1}, a_{2}\right) .
\end{aligned}
$$

Therefore

$\psi\left(y, t ; a_{1}, a_{2}, i \omega\right)=-\omega \int_{0}^{t} \sin [\omega(t-\tau)] \varphi\left(y, \tau ; a_{1}, a_{2}\right) \mathrm{d} \tau$ $+i \omega \int_{0}^{t} \cos [\omega(t-\tau)] \varphi\left(y, \tau ; a_{1}, a_{2}\right) \mathrm{d} \tau$.

For cosine oscillations of the plate

$$
\begin{aligned}
u_{c}(y, t)= & U_{1}(y, t)+U_{2}(y, t) \\
& -\omega \int_{0}^{t} \sin [\omega(t-\tau)] \varphi\left(y, \tau ; a_{1}, a_{2}\right) \mathrm{d} \tau,
\end{aligned}
$$

respectively, for sine oscillations of the plate

$$
\begin{aligned}
u_{s}(y, t)= & U_{1}(y, t)+U_{2}(y, t) \\
& +l \omega \int_{0}^{t} \cos [\omega(t-\tau)] \varphi\left(y, \tau ; a_{1}, a_{2}\right) \mathrm{d} \tau .
\end{aligned}
$$

2.4 Velocity field for ordinary second grade fluid $(\alpha \rightarrow 1=>\gamma \rightarrow \infty)$

In the case of a velocity field for an ordinary second grade fluid $(\alpha \rightarrow 1=>\gamma \rightarrow \infty)$, we obtain

$$
\begin{aligned}
& a_{1}=a_{2}=\frac{1}{\alpha_{2}}, b_{1}=0, b_{2}=-\frac{1-\operatorname{Pr}}{\alpha_{2} \operatorname{Pr}}, \\
& d_{1}=-\frac{\alpha_{2} \mathrm{Gr} \operatorname{Pr}}{(1-\operatorname{Pr})^{2}}, d_{2}=-\frac{\mathrm{Gr}}{(1-\operatorname{Pr})}, d_{3}=-d_{1},
\end{aligned}
$$

$\lim _{\gamma \rightarrow \infty} \varphi(y, t ; \operatorname{Pr} \gamma, \alpha \gamma)=\operatorname{erfc}\left(\frac{y \sqrt{\operatorname{Pr}}}{2 \sqrt{t}}\right)$ 
respectively,

$$
\begin{aligned}
& \lim _{\gamma \rightarrow \infty} \psi\left(y, t ; \operatorname{Pr} \gamma, \alpha \gamma,-b_{2}\right) \\
& \quad=-b_{2} \exp \left(-b_{2} t\right) * \varphi(y, t ; \operatorname{Pr}, 0),
\end{aligned}
$$

and we find the velocity profile,

$u(y, t)=U_{1}(y, t)+U_{2}(y, t)+\psi\left(y, t ; \frac{1}{\alpha_{2}}, \frac{1}{\alpha_{2}}, i \omega\right)$,

with

$$
\begin{aligned}
U_{1}(y, t)= & \varphi\left(y, t ; \frac{1}{\alpha_{2}}, \frac{1}{\alpha_{2}}\right)+\frac{\alpha_{2} \operatorname{Gr} \operatorname{Pr}}{1-\operatorname{Pr}} \\
& \times\left[b_{2} \exp \left(-b_{2} t\right) * \varphi(y, t ; \operatorname{Pr}, 0)\right. \\
& \left.+\psi\left(y, t ; \frac{1}{\alpha_{2}}, \frac{1}{\alpha_{2}},-b_{2}\right)\right], \\
U_{2}(y, t)= & \frac{\mathrm{Gr}}{1-\operatorname{Pr}} \int_{0}^{t} \\
& \times\left[\varphi(y, \tau ; \operatorname{Pr}, 0)-\varphi\left(y, \tau ; \frac{1}{\alpha_{2}}, \frac{1}{\alpha_{2}}\right)\right] \mathrm{d} \tau,
\end{aligned}
$$

where the notation $*$ represents the convolution product.

\section{Special cases}

3.1 Velocity field for the fractional Newtonian fluid $\left(\alpha_{2}=0,0<\alpha<1\right)$

In the case of a velocity field for the fractional Newtonian fluid $\left(\alpha_{2}=0,0<\alpha<1\right)$, we have $b_{1}=\frac{\mathrm{Gr}}{\gamma(1-\mathrm{Pr})}, b_{2}=$ $\alpha \gamma, a_{1}=\gamma, a_{2}=\alpha \gamma, d_{1}=\frac{\mathrm{Gr}}{\gamma(1-\mathrm{Pr})}, d_{2}=\frac{\alpha \mathrm{Gr}}{1-\operatorname{Pr}}, d_{3}=$ $0, \operatorname{Pr} \neq 1$, respectively,

$u(y, t)=U_{1}(y, t)+U_{2}(y, t)+\psi(y, t ; \gamma, \alpha \gamma, i \omega)$,

with

$U_{1}(y, t)=\left(1-d_{1}\right) \varphi(y, t ; \gamma, \alpha \gamma)+d_{1} \varphi(y, t ; \operatorname{Pr} \gamma, \alpha \gamma)$,

$U_{2}(y, t)=d_{2} \int_{0}^{t}[\varphi(y, \tau ; \operatorname{Pr} \gamma, \alpha \gamma)-\varphi(y, \tau ; \gamma, \alpha \gamma)] \mathrm{d} \tau$.
3.2 Velocity field for the ordinary Newtonian fluid

$$
\left(\alpha_{2}=0, \alpha=1\right)
$$

We use $\lim _{\gamma \rightarrow \infty} \varphi(y, t ; \gamma, \alpha \gamma)=\operatorname{erfc}\left(\frac{y}{2 \sqrt{t}}\right)$,

$U_{1}(y, t)=\operatorname{erfc}\left(\frac{y}{2 \sqrt{t}}\right)$,

$U_{2}(y, t)=\frac{\mathrm{Gr}}{1-\operatorname{Pr}} \int_{0}^{\int_{t}}\left[\operatorname{erfc}\left(\frac{y \sqrt{\operatorname{Pr}}}{2 \sqrt{\tau}}\right)-\operatorname{erfc}\left(\frac{y}{2 \sqrt{\tau}}\right)\right] \mathrm{d} \tau$.

The velocity field for the ordinary Newtonian fluid is

$$
\begin{gathered}
u(y, t)=\frac{\mathrm{Gr}}{1-\operatorname{Pr}} \\
\times\left(\begin{array}{c}
\left(t+\frac{\operatorname{Pr} y^{2}}{2}\right) \operatorname{erfc}\left(\frac{y \sqrt{\operatorname{Pr}}}{2 \sqrt{t}}\right)-\left(t+\frac{y^{2}}{2}\right) \operatorname{erfc}\left(\frac{y}{2 \sqrt{t}}\right) \\
+\frac{y \sqrt{t}}{\sqrt{\pi}}\left(\exp \left(-\frac{y^{2}}{4 t}\right)-\sqrt{\operatorname{Pr}} \exp \left(-\frac{\operatorname{Pr} y^{2}}{4 t}\right)\right) \\
+\frac{2}{\sqrt{\pi}} \int_{\frac{y}{2 \sqrt{t}}}^{\infty} \cos \left(\omega t-\frac{\omega y^{2}}{4 x^{2}}\right) \exp \left(-x^{2}\right) \mathrm{d} x \\
+i \frac{2}{\sqrt{\pi}} \int_{\frac{y}{2 \sqrt{t}}}^{\infty} \sin \left(\omega t-\frac{\omega y^{2}}{4 x^{2}}\right) \exp \left(-x^{2}\right) \mathrm{d} x
\end{array}\right) .
\end{gathered}
$$

\section{Numerical results and discussions}

In order to obtain some information on the fluid flow parameters, we have made several numerical simulations using Mathcad software. The results obtained are presented in the graphs from Figs. 1, 2, 3, 4, and 5. All the parameters and profiles are dimensionless.

We were interested, first, in an analysis of the influence of the fractional parameter $\alpha$ on the dimensionless temperature and the fluid flow velocity. Also, the influence of the Prandtl number $\operatorname{Pr}$ on the temperature and fluid velocity were studied. The influence of the Grashof number Gr for velocity is also presented. The ordinary fluid, corresponding to the unit fractional parameter, is compared with a few cases with the fractional parameter.

The diagrams of Fig. 1 are plotted in order to discuss the influence of the fractional parameter $\alpha$ on the fluid temperature. The curves corresponding to the temperature $\theta(y, t)$ are sketched versus $\mathrm{y}$, for different values of the time and of the fractional parameter $\alpha$, namely, $\alpha \in\{0.4,0.6,0.8,1.0\}$. For the Prandtl number we used the value $\operatorname{Pr}=5$.

By increasing values of the fractional parameter $\alpha$ the temperature increases. The thickness of the thermal boundary layer is increasing with the time $t$ and with the parameter $\alpha$. 

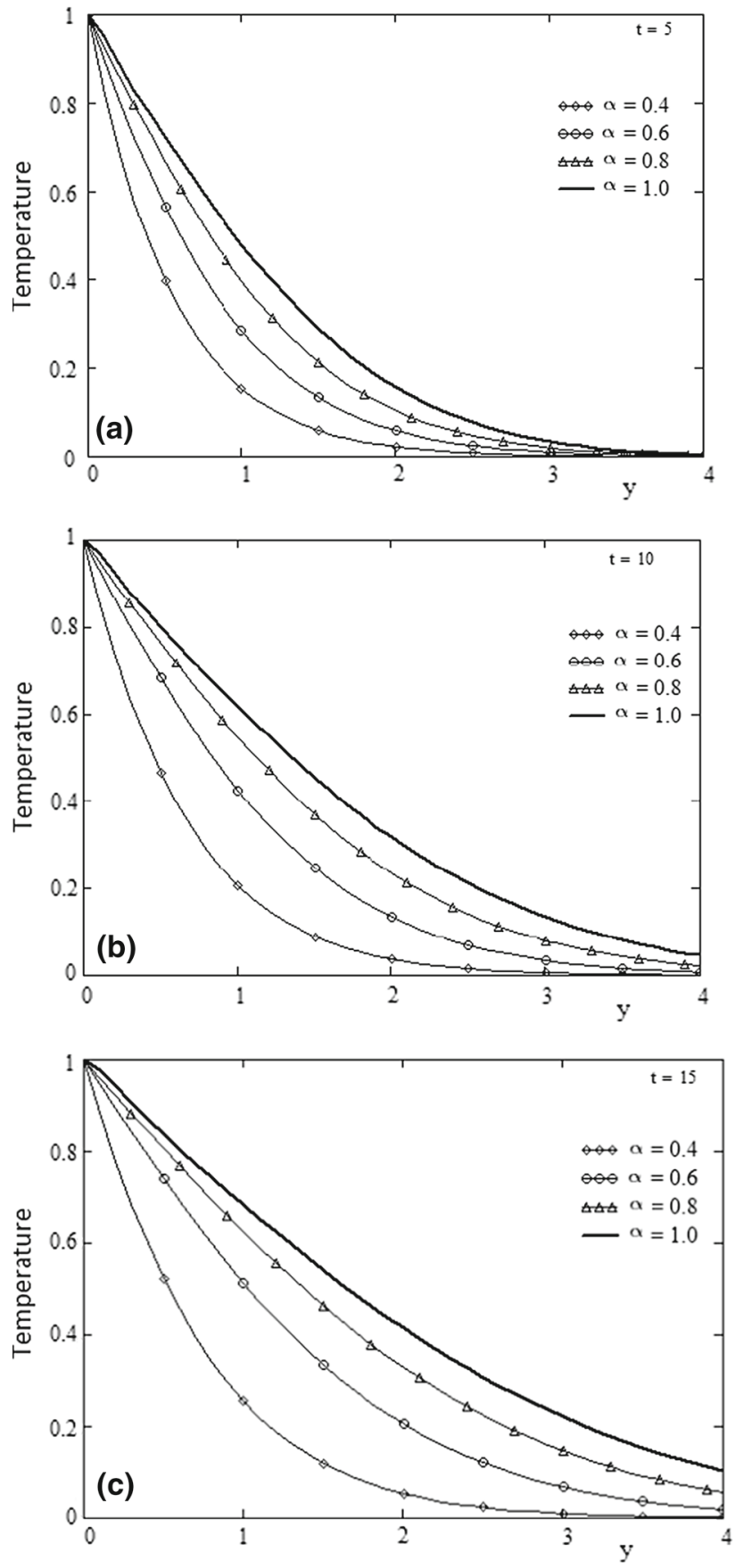

Fig. 1 Profiles of temperature versus $y$ for $\operatorname{Pr}=5$

The influence of the Prandtl number Pr on the fluid temperature is shown in Fig. 2. For the curves plotted in Fig. 2 , the fractional model corresponding to $\alpha=0.5$ was considered. It is observed from Fig. 2 that, at small values of the Prandtl number, the thermal diffusivity is large and the thickness of the thermal boundary layer decreases with the Prandtl number.

Figure 3 was drawn in order to analyze the influence of the fractional parameter $\alpha$ on the fluid velocity. Both cases of
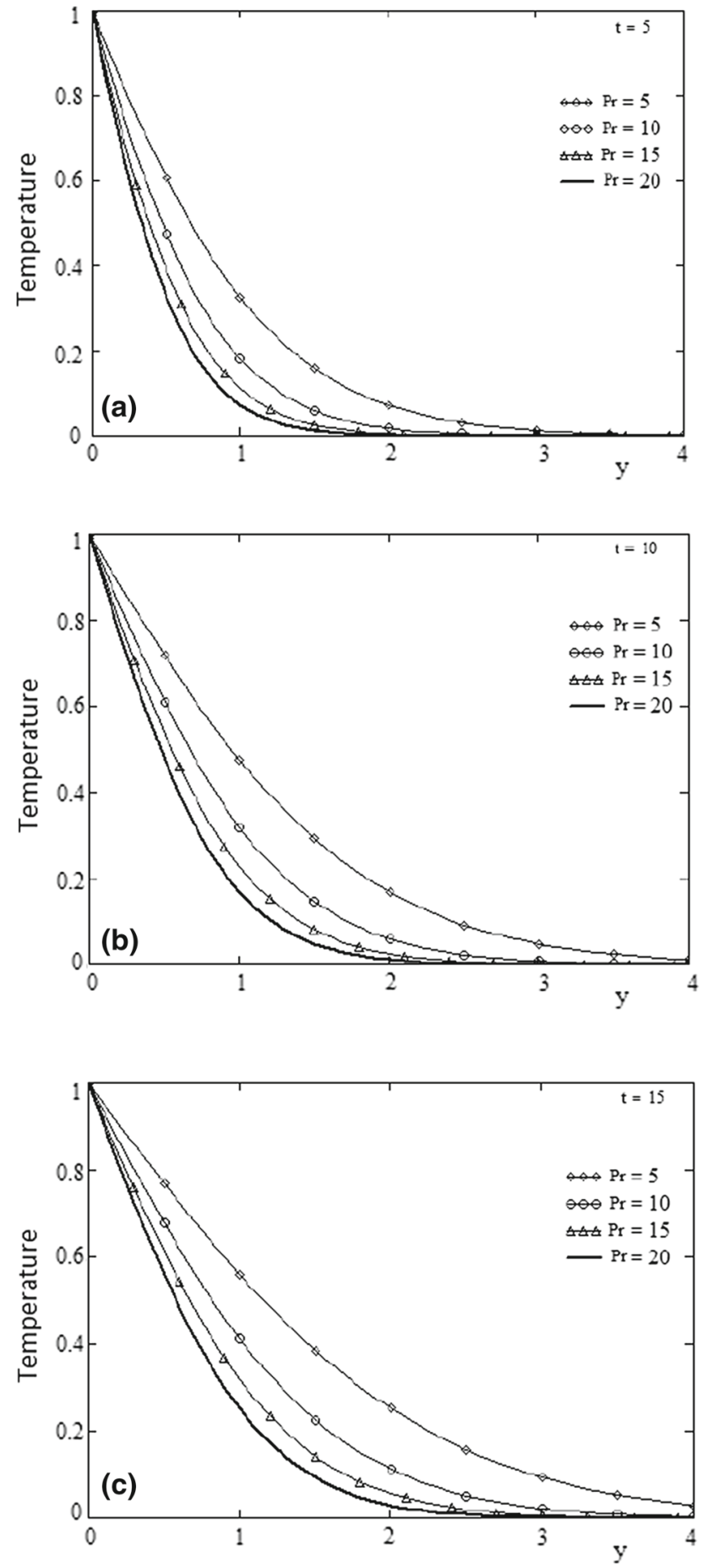

Fig. 2 Profiles of temperature versus $y$ for $\alpha=0.5$ and Pr variation for different values of $t$

the plate oscillations were considered, namely cosine oscillations and sine oscillations. For Fig. 3 we used the values $\operatorname{Pr}=5, \mathrm{Gr}=1.5, \alpha_{2}=0.5, \omega=2$. It must be emphasized that, if the fractional parameter $\alpha$ has low values, then the fluid velocity is higher. The ordinary fluid moves the slowest. 

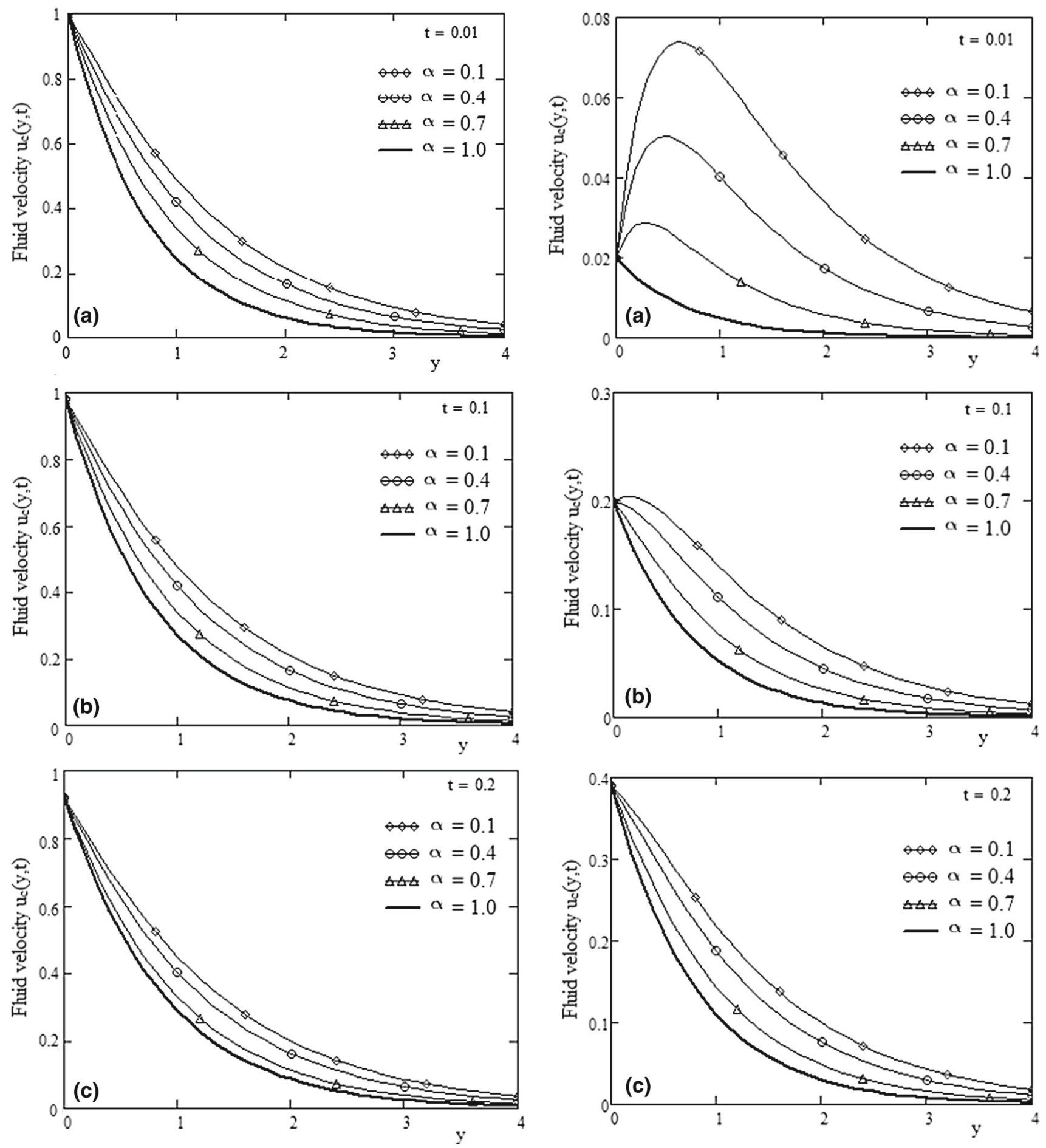

Fig. 3 Profiles of cosine and sine oscillation versus $y$ for $\alpha$ variation and different values of $t$

The diagrams of Fig. 4 are plotted in order to discuss the influence of the Prandtl number Pr on the fluid velocity. The curves corresponding to the fluid velocity are sketched versus $\mathrm{y}$, for different values of the time and of the Prandtl number $\operatorname{Pr}$, namely, $\operatorname{Pr} \in\{5,10,15,20\}$. For the other constants we used the values $\alpha=0,2, \omega=2, \mathrm{Gr}=10, \alpha_{2}=0.5$.
By increasing the value of the Prandtl number Pr, the velocity of the fluid for cosine and sine oscillation decreases and by increasing the time the influence of the cosine oscillation is very much smaller than in the case of the sine oscillation. 

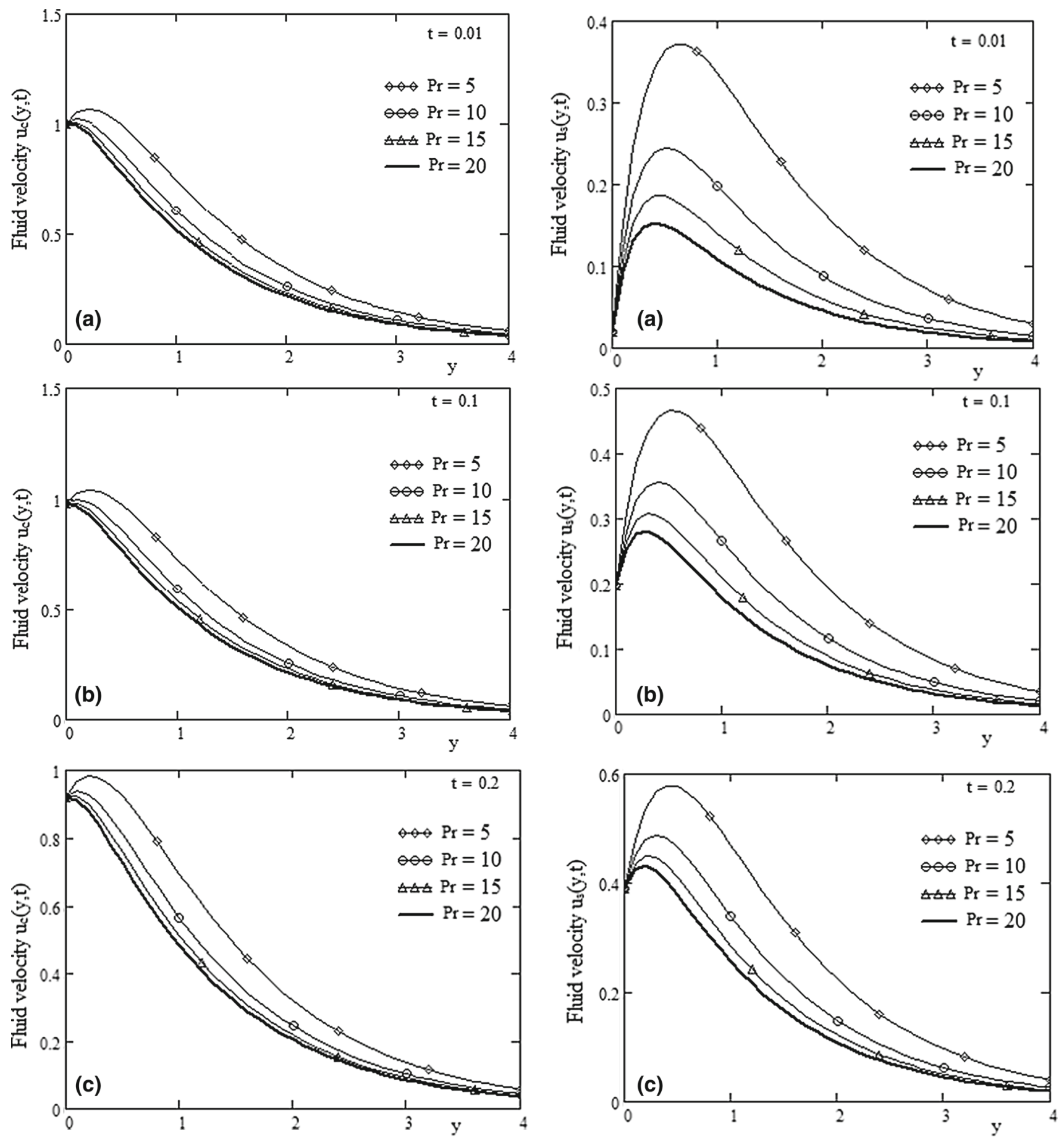

Fig. 4 Profiles of cosine and sine oscillation versus $y$ for Pr variation and different values of $t$

Figure 5 was plotted in order to discuss the influence of the Grashof number Gr on the fluid velocity. The curves corresponding to the fluid velocity are sketched versus y, for different values of the time and of the Grashof number Gr, namely $\operatorname{Gr} \in\{3,5,7,9\}$. For the other constants we used the values $\operatorname{Pr}=5, \quad \omega=2, \quad \alpha=0.5, \alpha_{2}=0.5$.
The curves show the behavior of the cosine and sine oscillations for the velocity. By increasing the value of the Grashof number $\mathrm{Gr}$ the velocity of the fluid for the cosine and sine oscillations increases and by increasing the time the influence of the cosine oscillation is very much smaller than in the case of the sine oscillation. In Fig. 6, the curves are plot- 

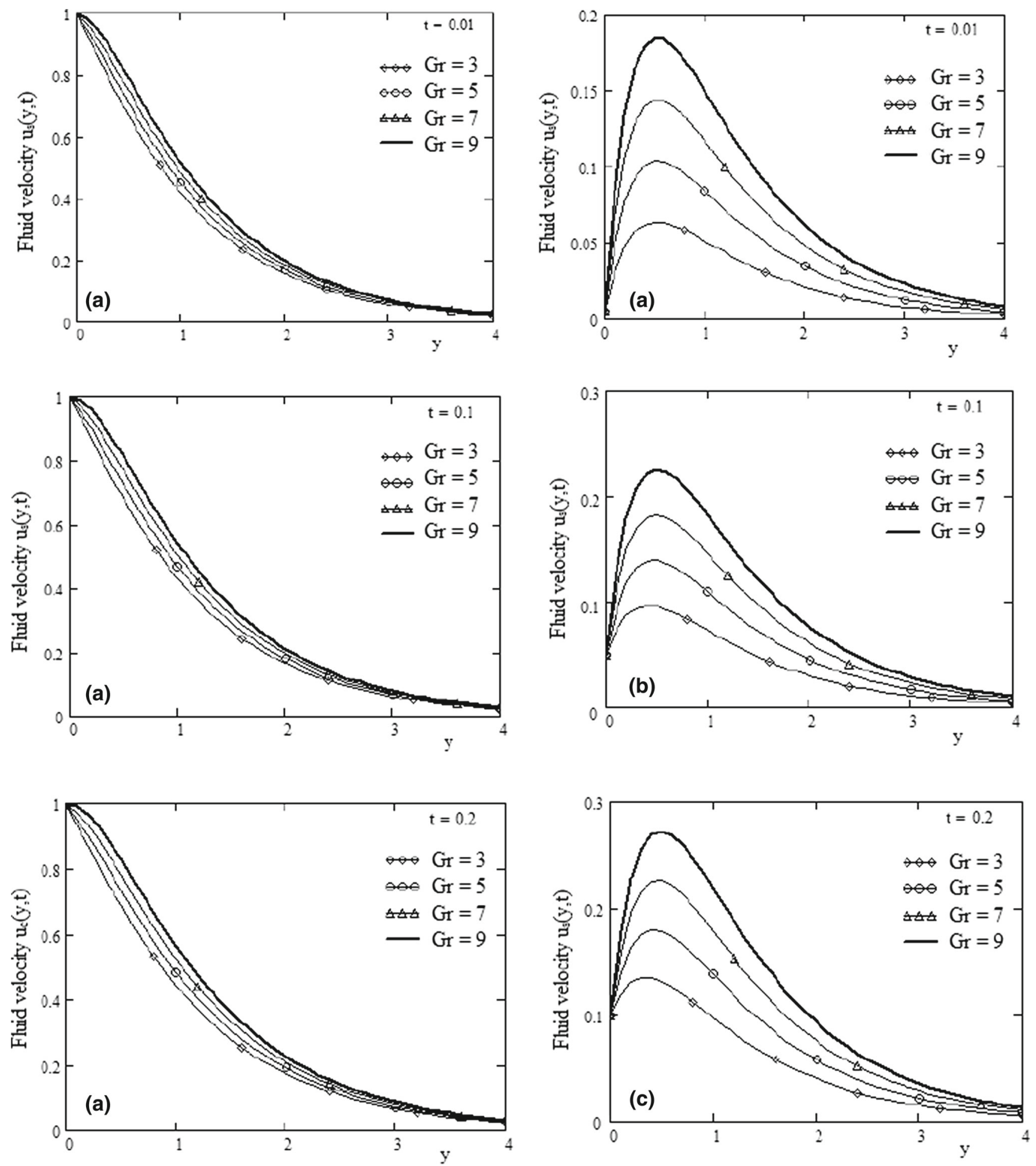

Fig. 5 Profiles of cosine and sine oscillation versus $y$ for Gr variation and different values of $t$

ted in order to show a comparison between second grade and Newtonian fluids for fractional and ordinary derivatives for the two cases of sine and cosine oscillations. The fractional fluids (both second grade and Newtonian) flow faster than ordinary fluids. The fractional second grade fluid shows the highest velocity, whereas the ordinary Newtonian fluid has minimum velocity. 

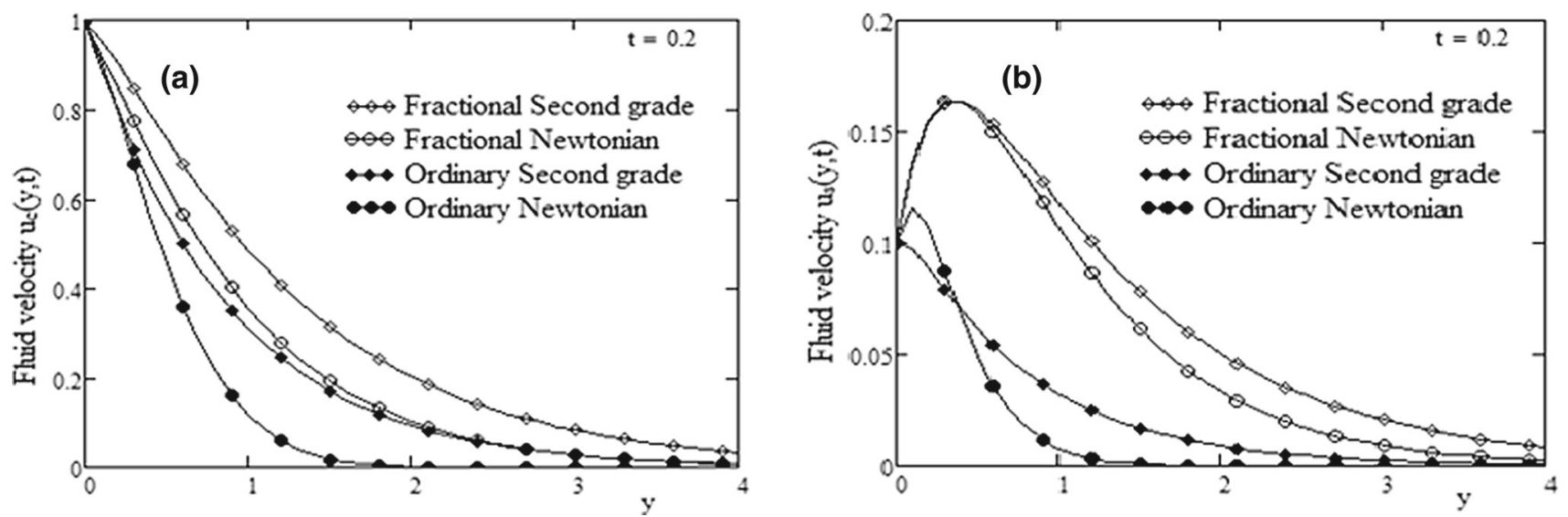

Fig. 6 Comparison of cosine (a) and sine (b) velocities for fractional second grade and Newtonian fluids with ordinary second grade and Newtonian fluids when $t=0.2$

\section{Conclusions}

The Caputo-Fabrizio fractional derivatives approach was used and the heat transfer analysis in a second grade fluid over an infinite oscillating vertical plate was studied. The solution of the problem in closed form was obtained using the Laplace transform method. Some limiting solutions were extracted corresponding to ordinary second grade and Newtonian fluids. The results were analyzed graphically for timefractional, second grade parameters, and Prandtl and Grashof numbers for various times. By the following remarks we conclude this article.

1. On increasing the fractional parameter, the temperature increases. The temperature is maximum for ordinary fluid $(\alpha=1)$.

2. The temperature decreases with increasing Prandtl number.

3. For large times, the thickness of the thermal boundary layer increases.

4. The velocity decreases with increasing fractional parameter; this behavior is quite opposite to that of the temperature.

5. The velocity decreases with increasing Prandtl number.

6. The velocity increases with increasing Grashof number.

7. The momentum boundary layer thickness of the cosine velocity is greater than the sine velocity.

8. Fractional fluids (second grade and Newtonian) have highest velocities. This shows that the fractional parameter enhances the fluid flow.

Open Access This article is distributed under the terms of the Creative Commons Attribution 4.0 International License (http://creativecomm ons.org/licenses/by/4.0/), which permits unrestricted use, distribution, and reproduction in any medium, provided you give appropriate credit to the original author(s) and the source, provide a link to the Creative Commons license, and indicate if changes were made. Funded by $\mathrm{SCOAP}^{3}$.

\section{Appendix}

We have

$$
\begin{aligned}
\Phi(y, s ; a, b) & =\frac{1}{s} \exp \left(-y \sqrt{\frac{a s}{s+b}}\right), \\
\varphi(y, t ; a, b) & =L^{-1}\{\Phi(y, s ; a, b)\} \\
& =1-\frac{2 a}{\pi} \int_{0}^{\infty} \frac{\sin (y x)}{x\left(a+x^{2}\right)} \exp \left(-\frac{b t x^{2}}{a+x^{2}}\right) \mathrm{d} x
\end{aligned}
$$

$$
\begin{aligned}
F(y, s ; a, b, c) & =\frac{1}{s-c} \exp \left(-y \sqrt{\frac{a s}{s+b}}\right) \\
& =\Phi(y, s ; a, b)+\psi(y, s ; a, b, c),
\end{aligned}
$$

$\psi(y, s ; a, b, c)=\frac{1}{s-c} \Phi(y, s ; a, b)$

$$
\begin{aligned}
\psi(y, t ; a, b, c)= & L^{-1}\{\psi(y, s ; a, b, c)\} \\
= & \exp \left(c t-y \sqrt{\frac{a c}{b+c}}\right) \\
& -1-\frac{2 a c}{\pi} \int_{0}^{\infty} \frac{\sin (y x)}{x\left[a c+(b+c) x^{2}\right]} \\
& \exp \left(-\frac{b t x^{2}}{a+x^{2}}\right), \\
f(y, t ; a, b, c)= & L^{-1}\{F(y, s ; a, b, c)\} \\
= & \Phi(y, t ; a, b)+\psi(y, t ; a, b, c),
\end{aligned}
$$


$G(y, s ; a, b)=\frac{1}{s^{2}} \exp \left(-y \sqrt{\frac{a s}{s+b}}\right)=\frac{1}{s} \Phi(y, s ; a, b)$,

$g(y, t ; a, b)=\int_{0}^{t} \varphi(y, \tau ; a, b) \mathrm{d} \tau$.

\section{References}

1. V. Kulish, J. Luis Lage, Application of fractional calculus to fluid mechanics, J. Fluids Engg. 124(3), 4 (2002). doi:10.1115/ 1.1478062

2. L. Debnath, Recent applications of fractional calculus to science and engineering. Int. J. Math. Math. Sci. 203(54), 3413-3442 (2003)

3. R. Hilfer, Threefold introduction to fractional derivatives. In Anomalous Transport (Wiley-VCH Verlag GmbH\&Co.KGaA, Germany, 2008)

4. R. Gorenflo, F. Mainardi, D. Moretti, P. Paradisi, Time fractional diffusion: a discret random walk approach. Nonlinear Dynam. 29, 129-143 (2002)

5. M. Caputo, M. Fabrizio, A new definition of fractional derivative without singular kernel. Progr. Fract. Differ. Appl. 1(2), 73-85 (2015)

6. W. Tan, X. Mingyu, Unsteady flows of a generalized second grade fluid with the fractional derivative model between two parallel plates. Acta. Mech. Sin. 20(5), 471-476 (2004)

7. C. Friedrich, Relaxation and retardation functions of the Maxwell model with fractional derivative. Rheol. Acta. 30, 151-158 (1991)

8. W.C. Tan, W.X. Pan, M.Y. Xu, A note on unsteady flows of a viscoelastic fluid with fractional Maxwell model between two parallel plates. Int. J. Nonlinear Mech. 38, 645-650 (2003)

9. T. Hayat, S. Nadeem, S. Asghar, Periodic unidirectional flows of a viscoelastic fluid with the fractional Maxwell model. Appl. Math. Comput. 151(1), 153-161 (2004)

10. Y. Yin, K.Q. Zhu, Oscillating flow of a viscoelastic fluid in a pipe with the fractional Maxwell model. Appl. Math. Comput. 173(1), 231-242 (2006)

11. H.T. Qi, H. Jin, Unsteady rotating flows of viscoelastic fluid with the fractional Maxwell model between coaxial cylinders. Acta. Mech. Sin. 22, 301-305 (2006)

12. H.T. Qi, M.Y. Xu, Unsteady flow of viscoelastic fluid with fractional Maxwell model in a channel. Mech. Res. Commun. 34, 210 221 (2007)

13. A. Mahmood, S. Parveen, A. Ara, N.A. Khan, Exact analytic solutions for the unsteady flow of a non-Newtonian fluid between two cylinders with fractional derivative model. Commun. Nonlinear Sci. Numer. Simul. 14(8), 3309-3319 (2009)

14. C. Fetecau, C. Fetecau, M. Jamil, A. Mahmood, Flow of fractional Maxwell fluid between coaxial cylinders. Arch. Appl. Mech. 81(8), 1153-1163 (2011)

15. M. Jamil, A. Rauf, A.A. Zafar, N.A. Khan, New exact analytical solutions for Stokes' first problem of Maxwell fluid with fractional derivative approach. Comput. Math. Appl. 62(3), 1013-1023 (2011)
16. M. Khan, T. Hayat, S. Asghar, Exact solutions for MHD flow of a generalized Oldroyd-B fluid with modified Darcy's law. Int. J. Eng. Sci. 44, 333-339 (2006)

17. M. Khan, K. Maqbool, T. Hayat, Influence of Hall current on the flows of a generalized Oldroyd-B fluid in a porous space. Acta Mech. 184, 1-13 (2006)

18. M. Khan, S. Hyder Ali, H. Qi, On accelerated flows of a viscoelastic fluid with the fractional Burgers' model. Nonlinear Anal. Real World Appl. 10(4), 2286-2296 (2009)

19. C. Fetecau, A. Mahmood, C. Fetecau, D. Vieru, Some exact solutions for the helical flow of a generalized Oldroyd-B fluid in a circular cylinder. Comput. Math. Appl. 56(12), 3096-3108 (2008)

20. H. Qi, H. Jin, Unsteady helical flows of a generalized Oldroyd-B fluid with fractional derivative. Nonlinear Anal. Real World Appl. 10(5), 2700-2708 (2009)

21. M. Jamil, N.A. Khan, A.A. Zafar, Translational flows of an Oldroyd-B fluid with fractional derivatives. Comput. Math. Appl. 62(3), 1540-1553 (2011)

22. L. Zheng, Y. Liu, X. Zhang, Slip effects on MHD flow of a generalized Oldroyd-B fluid with fractional derivative. Nonlinear Anal. Real World Appl. 13(2), 513-523 (2012)

23. Y. Liu, L. Zheng, X. Zhang, F. Zong, Some oscillation flows of a generalized Oldroyd-B fluid in uniform magnetic field. In Proceedings of the World Congress on Engineering, vol. 3, 1971-1974 (WCE' '10, London, 2010)

24. D. Tong, Starting solutions for oscillating motions of a generalized Burgers' fluid in cylindrical domains. Acta Mech. 214(3-4), 395$407(2010)$

25. L.C. Zheng, F.F. Zhao, X.X. Zhang, An exact solution for an unsteady flow of a generalized Burgers' fluid induced by an accelerating plate. Int. J. Nonlinear Sci. Num. Simul. 11(6), 457-464 (2011)

26. W.C. Tan, F. Xian, L. Wei, Exact solution for the unsteady Couette flow of the generalized second grade fluid. China Sci. Bull. 47, 1226-1228 (2002)

27. M. Khan, S. Nadeem, T. Hayat, A.M. Siddiqui, Unsteady motions of a generalized second grade fluid. Math. Comput. Model. 41, 629-637 (2005)

28. M. Khan, S. Hyder Ali, Q. Haitao, Exact solutions for some oscillating flows of a second grade fluid with a fractional derivative model. Math. Comput. Model. 49(7-8), 1519-1530 (2009)

29. M. Athar, M. Kamran, M. Imran, On the unsteady rotational flow of a fractional second grade fluid through a circular cylinder. Meccanica 81(11), 1659-1666 (2011)

30. D. Vieru, C. Fetecau, C. Fetecau, Time fractional free convection flow near a vertical plate with Newtonian heating and mass discussion. Thermal Sci. 19, S85-S98 (2015)

31. F. Ali, I. Khan, S. Shafie, Closed form solutions for unsteady free convection flow of a second grade fluid over an oscillating vertical plate. PLoS One 9(2), e85099 (2014). doi:10.1371/journal.pone. 0085099

32. I.S. Gradshteyn, I.M. Ryzhik, Table of integral, series and products, 8th edn. (Academic Press, USA, 2014) 